\title{
Characterization of the Skew-Normal Distribution Via Order Statistics and Record Values
}

\author{
M. Gharib ${ }^{1}$, M.M. Mohie El-Din ${ }^{2} \&$ A. Sharawy ${ }^{3}$ \\ ${ }^{1}$ Department of Mathematics, Faculty of Science, Ain Shams University, Cairo, Egypt \\ ${ }^{2}$ Department of Mathematics, Faculty of Science (men), Al-Azhar University, Cairo, Egypt \\ ${ }^{3}$ Department of Mathematics, Al-Safwa High Institute of Engineering, Cairo, Egypt \\ Correspondence: A. Sharawy, Department of Mathematics, Al-Safwa High Institute of Engineering, Cairo, Egypt. \\ E-mail: mrali112@yahoo.com
}

Received: November 12, 2014

Accepted: November 27, 2014 Online Published: December 22, 2014

doi:10.5539/ijsp.v4n1p59

URL: http://dx.doi.org/10.5539/ijsp.v4n1p59

\begin{abstract}
Characterization of a probability distribution is the investigation of those unique properties enjoyed by that distribution. In this article the general condition moments technique is used to obtain some new characterization results for the skew-normal distribution based on the order statistics as well as the record values of a random sample drawn from this distribution. The achieved results can be used to improve fitting and modeling skew data using the skew-normal distribution. Further, the new results are specialized to the standard normal distribution.
\end{abstract}

Keywords: Characterization, order statistics, record values, conditional moment, skew-normal distribution

\section{Introduction}

Azzalini (1985, 1986) have introduced the skew-normal distribution which includes the normal distribution and has some properties like the normal and yet is skew. This class of distributions is useful in studying robustness and modelling skewness. Since the normal distribution is still the most commonly used distribution both in statistical theory and applications, then a family of distributions like the skew normal one that possesses the same properties of the normal family have a great potential impact in the theoretical and applied probability and statistics. Despite of this potential impact there are relatively few statisticians who used this family in their theoretical and applied works.

It is well known that characterization theorems represent an indispensable tool for understanding the missing links between the mathematical structure of statistical distributions and the actual behavior of real world random phenomena. It is reasonable to assert that although the characterization theory does not offer a well-defined method for solving real world problems, it guides the statistician as the case may be toward a proper scientific solution.

In fact, research on characterization for the skew-normal distribution is still in its early stage. Characterizations concerning the skew normal distribution are given by Gupta and Wen-Jang ( 2002), Gupta and Jose (2004) and Gharib et al. (2014). In this article the general condition moment technique is used to obtain some new characterization results for the skew-normal distribution based on the order statistics as well as the record values of a random sample drawn from this distribution. The achieved results can be used to improve fitting and modeling skew data using the skew-normal distribution. Further, the new results are specialized to the standard normal distribution.

A random variable (rv) $\mathrm{Z}$ has a skew-normal distribution with parameter $\lambda$, denoted by $\mathrm{Z} \sim \mathrm{SN}(\lambda)$ if its probability density function (pdf) is given by:

$$
f(z, \lambda)=2 \phi(z) \Phi(\lambda z)
$$

where, $\phi$ and $\Phi$ are, respectively, the probability density function and cumulative distribution function of the standard normal distribution, and $z$ and $\lambda$ are real numbers Azzalini (1985).

The cumulative distribution function (cdf) corresponding to (1.1) is given by 


$$
F(z, \lambda)=\int_{-\infty}^{z} 2 \phi(u) \Phi(\lambda u) d u
$$

For the structural and other properties of the skew-normal distribution see Brown (2001) and the references therein.

The following lemma is needed in proving the results of the article.

\section{Lemma}

If $\mathrm{X}_{1: \mathrm{n}}, \mathrm{X}_{2: \mathrm{n}}, \ldots, \mathrm{X}_{\mathrm{n}: \mathrm{n}}$, are the order statistics (os) of a random sample of size (n) drawn from a continuous distribution with cdf $F($.$) then the conditional joint distribution of the os \mathrm{X}_{1: \mathrm{n}}, \ldots, \mathrm{X}_{\mathrm{k}-1 \mathrm{n}}$ given $\mathrm{X}_{\mathrm{k}: \mathrm{n}}=\mathrm{x}$ is identical to the joint distribution of the os $Y_{1: k-1}^{x}, Y_{2: k-1}^{x}, \ldots, Y_{k-1: k-1}^{x}$ from a random sample of size $(k-1)$ with cdf $H_{x}(u)$ having the form:

$$
H_{x}(u)= \begin{cases}F(u) / F(x) & u<x \\ 1 & u \geq x\end{cases}
$$

and the conditional joint distribution of the os $X_{k+1: n}, X_{k+2: n}, \ldots, X_{n: n}$, given $X_{k: n}=x$, is identical to the joint distribution of the os $Z_{1: n-k}^{x}, Z_{2: n-k}^{x}, \ldots, Z_{n-k-n-k}^{x}$ from a random sample of size $(n-k)$ with cdf $R_{x}(u)$ having the form:

$$
R_{x}(u)= \begin{cases}{[F(u)-F(x)] / \bar{F}(x)} & u>x \\ 0 & u \leq x\end{cases}
$$

where $\bar{F}(x)=1-F(x)$.

This lemma is proved in Arnold et al. (2008).

Using the above lemma, we have:

$$
\begin{gathered}
E\left(X_{1: n}+\cdots+X_{k-1: n} \mid X_{k: n}=x\right)=E\left(Y_{1: k-1}^{x}+\cdots+Y_{k-1: k-1}^{x}\right)=(k-1) E\left(Y_{1}^{x}\right) \\
=(k-1)[F(x)]^{-1} \int_{-\infty}^{x} u f(u) d u
\end{gathered}
$$

and:

$$
\begin{gathered}
E\left(X_{k-1: n}+\cdots+X_{n: n} \mid X_{k: n}=x\right)=E\left(Z_{1: n-k}^{x}+\cdots+Z_{n-k: n-k}^{x}\right)=(n-k) E\left(Z_{1}^{x}\right) \\
=(n-k)[\bar{F}(x)]^{-1} \int_{x}^{\infty} u f(u) d u .
\end{gathered}
$$

Further from (1.5) and (1.6), we readily obtain:

$$
E\left(X_{1: n}+\cdots+X_{n: n} \mid X_{k: n}=x\right)=x+\frac{(k-1)}{F(x)} \int_{-\infty}^{x} u f(u) d u+\frac{(n-k)}{\bar{F}(x)} \int_{x}^{\infty} u f(u) d u .
$$

\section{Main Results}

\subsection{Characterization via General Conditional Moments of Order Statistics}

The problem of characterizing distributions through conditional moments of order statistics has been of increasing interest during the last few decades due to its several applications, for example, the study of ( $n-$ $r)$-out-of-n systems. A $(n-r)$-out-of-n systems consists of $(n)$ independent and identically distributed components, and it works as long as at least $(n-r)$ components are working. If $X_{i}$ represents the lifetime of the $i^{\text {th }}$ component, $i=1,2, \ldots, n$, the survival function of the $(n-r)$-out-of-n system is the same as that of the $(r+1)^{t h}$ os $X_{r+1, n}$ from this sample of $(n)$ random variables. Hence the results obtained for os hold for $(n-$ $r$ )-out-of-n systems, so characterizing distributions using os plays an important role in reliability theory.

Theorems 2.1-2.4 below characterizes the skew-normal distribution using conditional moments of os.

\section{Theorem 2.1}

Let $X$ be a continuous rv with pdf $f($.$) , cdf F($.$) , survival function \bar{F}(\cdot)$, failure rate function $h($.$) and finite$ 
mean $\mu$. Let $\mathrm{X}_{1: \mathrm{n}} \leq \mathrm{X}_{2: \mathrm{n}} \leq \ldots \leq \mathrm{X}_{\mathrm{n}: \mathrm{n}}$ be the os of a random sample of size $n$ drawn from $X$. Suppose that $F($.) has a continuous second order derivative on $(-\infty, \infty)$. Then $X$ has a skew normal distribution $\operatorname{SN}(\lambda)$ with mean $\mu$ if and only if, for $k=1,2, \ldots, n$ :

$$
E\left(X_{k+1: n}+\cdots+X_{n: n} \mid X_{k: n}=y\right)=(n-k)\left\{h(y, \lambda)+\mu(\lambda) \bar{\Phi}\left(y \sqrt{1+\lambda^{2}}\right)[\bar{F}(y, \lambda)]^{-1}\right\},
$$

where $\bar{\Phi}(x)=1-\Phi(x)$ and $\mathrm{h}(x, \lambda)=\mathrm{f}(x, \lambda) / \bar{F}(x, \lambda)$.

\section{Theorem 2.2}

Let $X$ be a continuous rv with pdf $f($.$) , cdf F($.$) and finite mean \mu$ and let $X_{1: n} \leq X_{2: n} \leq \ldots \leq X_{n: n}$ be the os of a random sample of size $n$ drawn from $X$. Let $F($.$) has a continuous second order derivative on (-\infty, \infty)$. Then $X$ has a skew-normal distribution $\operatorname{SN}(\lambda)$ with finite mean $\mu$ if and only if:

$$
E\left(X_{1: n}+\cdots+X_{k-1: n} \mid X_{k: n}=y\right)=(k-1)\left[\mu(\lambda) \Phi\left(y \sqrt{1+\lambda^{2}}\right)-f(y, \lambda)\right][F(y, \lambda)]^{-1} .
$$

\section{Theorem 2.3}

Let $X$ be a continuous rv with pdf $f($.$) , cdf F($.$) , survival function \bar{F}($.$) and finite mean \mu$ and let $\mathrm{X}_{1: \mathrm{n}} \leq \ldots \leq \mathrm{X}_{\mathrm{n}: \mathrm{n}}$ be the os of a random sample of size $n$ drawn from $X$. Let $F($.$) has a continuous second$ order derivative on $(-\infty, \infty)$. Then $X$ has a skew-normal distribution $\operatorname{SN}(\lambda)$ with finite mean $\mu$ if and only if:

$$
E\left(S_{k}-\bar{S}_{k} \mid X_{k: n}=y\right)=y+\frac{m}{F(y, \lambda) \bar{F}(y, \lambda)}\left[\mu(\lambda) \Phi\left(y \sqrt{1+\lambda^{2}}\right)-f(y, \lambda)-\mu F(y, \lambda)\right],
$$

where

$$
S_{k}=X_{1: n}+\cdots+X_{k: n}, \bar{S}_{k}=X_{k+1: n}+\cdots+X_{n: n}, \mathrm{n}=2 \mathrm{~m}+1, \quad \mathrm{k}=\mathrm{m}+1 \text { and } \mathrm{m}=1,2,3, \ldots .
$$

\section{Theorem 2.4}

Let $X$ be a continuous rv with pdf $f($.$) , cdf F($.$) , survival function \bar{F}($.$) and nite mean \mu$ and let $\mathrm{X}_{1: \mathrm{n}}$ $\leq \ldots \leq \mathrm{X}_{\mathrm{n}: \mathrm{n}}$ be the os of a random sample of size $n$ drawn from $X$. Let $F($.$) has a continuous second order$ derivative on $(-\infty, \infty)$. Then $X$ has a skew-normal distribution $\operatorname{SN}(\lambda)$ with finite mean $\mu$ if and only if:

$$
E\left(T_{k} \mid X_{k: n}=y\right)=y+\mu(\lambda) m[\bar{F}(y, \lambda)]^{-1}+\frac{m[2 F(y, \lambda)-1]}{F(y, \lambda) \bar{F}(y, \lambda)}\left[f(y, \lambda)-\mu(\lambda) \Phi\left(y \sqrt{1+\lambda^{2}}\right)\right],
$$

where $T_{k}=X_{1: n}+\cdots+X_{n: n} \quad, \quad \mathrm{n}=2 \mathrm{~m}+1, \mathrm{k}=\mathrm{m}+1$ and $\mathrm{m}=1,2,3, \ldots$.

\subsection{Characterization via General Conditional Moments of Record Values}

Record values are found in many situations of daily life as well as in many situations applications suchas reliability theory (for comprehensive accounts of the theory and applications of record values see Arnold et al. (1998), Ahsanullah (1995), Ahsanullah (2004) and Ahsanullah et al. (2006). Characterizing the distributions via their record statistics has a long history, for excellent review one may refer to Shawky et al. (2006) Shawky et al. (2008) and Shawky et al. (2008b) among others. Suppose that $\left(X_{n}\right)_{n \geq 1}$ is a sequence of independent and identically distribution rvs. Let $Y_{n}=\max \left\{X_{j} \mid 1 \leq j \leq n\right\}$ for $n \geq 1$. We say $X_{j}$ is an upper record value of $\left\{X_{n} \mid n\right.$ $\geq 1\}$, if $Y_{j}>Y_{j-1}, j>1$. By definition $X_{1}$ is an upper record value. The indices at which the upper record values occur are given by the record times $\{\mathrm{U}(\mathrm{n}) ; \mathrm{n}>1\}$, where: $U(n)=\min \left\{j \mid j>U(n-1), X_{j}>X_{U(n-1)}, n>1\right\}$ and $\mathrm{U}(1)=1$.

Theorems 2.5 and 2.6 below characterize the skew-normal distribution using conditional moments of upper record values.

\section{Theorem 2.5}

Let $X$ be a continuous rv with pdf $f($.$) , cdf F($.$) , survival function \bar{F}($.$) and failure rate h($.$) . Assume that$ $F($.$) has a continuous second order derivative on (-\infty, \infty)$. and that $\mathrm{E}\left(\mathrm{X}_{\mathrm{U}(\mathrm{n}+1)}\right)$ is finite. Then $X$ has a skew-normal distribution $\mathrm{SN}(\lambda)$ if and only if, for $k=1,2, \ldots$

$$
E\left(X_{U(n+1)}^{2 k} \mid X_{U(n)}=y\right)=(2 k-1) ! !+\sum_{i=1}^{k} \frac{(2 k-1) ! !}{(2 i-1) ! !}\left\{\left[y^{2 i-1} h(y, \lambda)\right]+\mu(\lambda) \alpha_{i}(y, \lambda) / \bar{F}(y, \lambda)\right\},
$$


where $\quad \alpha_{i}(y, \lambda)=\sum_{j=1}^{i} \frac{(2 i-1) ! !}{(2 j-1) ! !} \frac{\left[y^{2 i-2} \phi\left(y \sqrt{1+\lambda^{2}}\right)\right]}{\left[1+\lambda^{2}\right]^{i-j+1 / 2}}, \quad \mathrm{i}=1,2 \ldots, \mathrm{k}, \quad \mathrm{j}=1,2 \ldots, \mathrm{i}$,

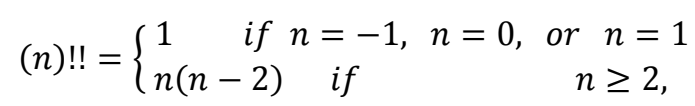

and (see Arfken (1985)).

\section{Special Case}

For $k=1,(2.5)$ reduces to:

$$
E\left(X_{U(n+1)}^{2} \mid X_{U(n)}=y\right)=1+y h(y, \lambda)+\mu(\lambda) \phi\left(y \sqrt{1+\lambda^{2}}\right) / \bar{F}(y, \lambda),
$$

\section{Theorem 2.6}

Let $X$ be a continuous rv with pdf $f($.$) , cdf F($.$) , survival function \bar{F}($.$) and failure rate h($.$) . Assume$ that $F($.$) has a continuous second order derivative on (-\infty, \infty)$ and that $\mathrm{E}\left(\mathrm{X}_{\mathrm{U}(\mathrm{n}+1)}\right)$ is finite. Then $X$ has a skew-normal distribution $\mathrm{SN}(\lambda)$ if and only if, for $k=0,1,2, \ldots$

$$
\begin{gathered}
E\left(X_{U(n+1)}^{2 k+1} \mid X_{U(n)}=y\right)=(2 k-1) ! ! \\
+\sum_{i=0}^{k} \frac{(2 k) ! !}{(2 i) ! !}\left\{\left[y^{2 i} h(y, \lambda)\right]+\mu(\lambda)[\bar{F}(y, \lambda)]^{-1}\left[\gamma_{i}(y, \lambda)+\beta_{i}(y, \lambda)\right]\right\},
\end{gathered}
$$

where $\beta_{i}(y, \lambda)=\frac{(2 i-1) ! !}{\left(1+\lambda^{2}\right)^{i}}\left[\bar{\Phi} \sqrt{1+\lambda^{2}}\right], i=0,1,2, \ldots, k$,

$$
\gamma_{i}(y, \lambda)=\sum_{j=1}^{i} \frac{(2 i-1) ! !}{(2 j-1) ! !}\left\{\left[y^{2 i-1} \phi\left(y \sqrt{1+\lambda^{2}}\right)\right] /\left[1+\lambda^{2}\right]^{i-j+1 / 2}\right\} \quad \text { and } \quad \mathrm{j}=1,2 \ldots, \mathrm{i} .
$$

\section{Special Case}

For $\mathrm{k}=0,(2.7)$ reduces to:

$$
E\left(X_{U(n+1)} \mid X_{U(n)}=y\right)=h(y, \lambda)+\mu(\lambda)[\bar{F}(y, \lambda)]^{-1} \bar{\Phi}\left(y \sqrt{1+\lambda^{2}}\right) .
$$

\section{Proofs of the Theorems}

\section{Proof of Theorem 2.1}

\section{Necessity:}

Suppose that $X \sim \mathrm{SN}(\lambda)$ with pdf and cdf given by (1.1) and (1.2).

Then using (1.6); we have

$$
E\left(X_{k+1: n}+\cdots+X_{n: n} \mid X_{k: n}=y\right)=2(n-k)[\bar{F}(y, \lambda)]^{-1} \int_{y}^{\infty} x \phi(x) \Phi(\lambda x) d x .
$$

On integrating by parts we get:

$$
E\left(X_{k+1: n}+\cdots+X_{n: n} \mid X_{k: n}=y\right)=2(n-k)[\bar{F}(y, \lambda)]^{-1}\left[\phi(y) \Phi(\lambda y)+\lambda \int_{y}^{\infty} \phi(x) \phi(\lambda x) d x\right] .
$$

Further:

$$
2 \lambda \int_{y}^{\infty} \phi(x) \phi(\lambda x) d x=\mu(\lambda) \Phi\left(y \sqrt{1+\lambda^{2}}\right) .
$$

Finally, recalling that $\mathrm{h}(\mathrm{y}, \lambda)=\mathrm{f}(\mathrm{y}, \lambda) / \bar{F}(\mathrm{y}, \lambda)$ and $f(y, \lambda)=2 \phi(y) \Phi(\lambda y)$ we get (2.1) from (3.1) and (3.2).

\section{Sufficiency:}


Suppose that $\mathrm{X}$ is a continuous rv with pdf $f($.$) , cdf F($.$) , finite mean \mu$ and $\mathrm{F}($.$) has a continuous second order$ derivative on $(-\infty, \infty)$. Suppose, also, that (2.1) is true. Hence, from (1.6) and (2.1) one can write:

$$
\int_{y}^{\infty} x f(x, \lambda) d x=f(y, \lambda)+\mu(\lambda) \bar{\Phi}\left(y \sqrt{1+\lambda^{2}}\right) .
$$

Differentiating both sides in this equation with respect to y, we obtain:

$$
\frac{d}{d y} f(y, \lambda)+y f(y, \lambda)=\mu(\lambda) \sqrt{2 \pi} \sqrt{1+\lambda^{2}} \phi(y) \phi(\lambda y) .
$$

Which is a linear first order differential equation in the unknown function $f(y)$.

Its solution is:

$$
f(y, \lambda)=\frac{\mu}{\lambda} \sqrt{\pi / 2} \sqrt{1+\lambda^{2}}[2 \phi(y) \Phi(\lambda y)]
$$

Now, the normalizing condition:

$$
\int_{-\infty}^{\infty} f(y, \lambda) d y=1 \quad \text { gives } \quad \mu(\lambda)=\sqrt{\frac{2}{\pi}}\left[\lambda / \sqrt{1+\lambda^{2}}\right] .
$$

Hence:

$$
f(y, \lambda)=2 \phi(y) \Phi(\lambda y) .
$$

Which is the pdf of the skew-normal distribution given by (1.1). This completes the proof of Theorem 2.1.

\section{Proof of Theorem 2.2}

The proof is obtained by repeating word-to-word the steps of the proof of Theorem 2.1.

\section{Proof of Theorem 2.3}

\section{Necessity:}

Suppose that $X \sim \mathrm{SN}(\lambda)$ with pdf and cdf given by (1.1) and (1.2) respectively. Then using (1.5) and (1.6), we have:

$$
\begin{gathered}
E\left(S_{k}-\bar{S}_{k} \mid X_{k: n}=y\right)=y+\frac{m}{F(y, \lambda)} \int_{-\infty}^{y} x f(x, \lambda) d x-\frac{m}{\bar{F}(y, \lambda)} \int_{y}^{\infty} x f(x, \lambda) d x= \\
y+\frac{m}{k-1} E\left(X_{1: n}+\cdots+X_{k-1: n} \mid X_{k: n}=y\right)-\frac{m}{n-k} E\left(X_{k+1: n}+\cdots+X_{n: n} \mid X_{k: n}=y\right) .
\end{gathered}
$$

The rest of the proof of necessity is obtained by applying (2.1) and (2.2).

\section{Sufficiency:}

Suppose that $X$ is a continuous rv with pdf $f($.$) , cdf F($.$) , finite mean \mu$ and $F($.$) has a continuous second$ order derivative on $(-\infty, \infty)$. Suppose, also, that (2.3) is true. Hence, using (2.1), (2.2) and (2.3) one can get:

$$
\begin{gathered}
\bar{F}(y, \lambda) \int_{-\infty}^{y} x f(x, \lambda) d x-F(y, \lambda) \int_{y}^{\infty} x f(x, \lambda) d x= \\
\mu(\lambda) \Phi\left(y \sqrt{1+\lambda^{2}}\right)-f(y, \lambda)-\mu(\lambda) F(y, \lambda) .
\end{gathered}
$$

Now, it is easy to see that:

$$
\bar{F}(y, \lambda) \int_{-\infty}^{y} x f(x, \lambda) d x-F(y, \lambda) \int_{y}^{\infty} x f(x, \lambda) d x=\int_{-\infty}^{y} x f(x, \lambda) d x-\mu(\lambda) F(y, \lambda) .
$$

Hence (3.6) reduces to: 


$$
\int_{-\infty}^{y} x f(x, \lambda) d x=\mu(\lambda) \Phi\left(y \sqrt{1+\lambda^{2}}\right)-f(y, \lambda) .
$$

Differentiating both sides of this equation with respect to $\mathrm{y}$, we get:

$$
\frac{d}{d y} f(y, \lambda)+y f(y, \lambda)=\mu(\lambda) \sqrt{2 \pi} \sqrt{1+\lambda^{2}} \phi(y) \phi(\lambda y) .
$$

Which is Equation (3.3) and thus having the solution given by (3.4). This completes the proof of Theorem 2.3.

\section{Proof of Theorem 2.4}

The proof is obtained by repeating word-to-word the steps of the proof of Theorem 2.3.

\section{Proof of Theorem 2.5:}

\section{Necessity:}

Suppose that $X \sim \mathrm{SN}(\lambda)$ with pdf and cdf given by (1.1) and (1.2), respectively.

Then we have:

$$
E\left(X_{U(n+1)}^{2 k} \mid X_{U(n)}=y\right)=\frac{2}{\bar{F}(y, \lambda)} \int_{y}^{\infty} u^{2 k} \phi(u) \Phi(\lambda u) d u=\frac{2}{\bar{F}(y, \lambda)} \int_{y}^{\infty} u^{2 k-1} \Phi(\lambda u) d \phi(u) .
$$

Now, repeated routine integration by parts gives:

$$
\begin{gathered}
-2 \int_{y}^{\infty} u^{2 k-1} \Phi(\lambda u) d \phi(u)=(2 k-1) ! ! \\
+\sum_{i=1}^{k} \frac{(2 k-1) ! !}{(2 i-1) ! !}\left\{\left[y^{2 i-1} f(y, \lambda)\right]+2 \lambda \int_{y}^{\infty} u^{2 i-1} \phi(u) \phi(\lambda u) d u\right\} .
\end{gathered}
$$

Again, repeated integration by parts gives:

$$
\begin{gathered}
2 \lambda \int_{y}^{\infty} u^{2 i-1} \phi(u) \phi(\lambda u) d u=\frac{\lambda \sqrt{2 / \pi}}{\left[1+\lambda^{2}\right]^{i-j+1}} \sum_{j=1}^{i} \frac{(2 i-2) ! !}{(2 j-2) ! !}\left[y^{2 i-2} \phi\left(y \sqrt{1+\lambda^{2}}\right)\right] \\
=\mu(\lambda) \alpha_{i}(y, \lambda) .
\end{gathered}
$$

Finally from (3.7), (3.8) and (3.9) we get (2.5).

\section{Sufficiency:}

Suppose that $X$ is a continuous rv with pdf $f($.$) and \operatorname{cdf} F($.$) and that F($.$) has a continuous second order$ derivative on $(-\infty, \infty)$. Suppose, also, that $\mathrm{E}\left(\mathrm{X}_{\mathrm{U}(\mathrm{n}+1)}\right)$ is finite and (2.5) is true. Then we have:

$$
\begin{gathered}
E\left(X_{U(n+1)}^{2 k} \mid X_{U(n)}=y\right)=[\bar{F}(y, \lambda)]^{-1} \int_{y}^{\infty} x^{2 k} f(x, \lambda) d x \\
=(2 k-1) ! !+\sum_{i=1}^{k} \frac{(2 k-1) ! !}{(2 i-1) ! !}\left\{\left[y^{2 i-1} h(y, \lambda)\right]+\frac{\mu(\lambda)}{\bar{F}(y, \lambda)} \alpha_{i}(y, \lambda)\right\} .
\end{gathered}
$$

Multiplying both sides of this equation by $\bar{F}(\mathrm{y})$ and then differentiating it with respect to y, we obtain:

$$
\begin{gathered}
-y^{2 i} f(y, \lambda)=-(2 k-1) ! ! f(y, \lambda)+\sum_{i=1}^{k} \frac{(2 k-1) ! !}{(2 i-1) ! !}(2 i-1) y^{2 i-2} f(y, \lambda)+ \\
+\sum_{i=1}^{k} \frac{(2 k-1) ! !}{(2 i-1) ! !}\left[y^{2 i-1} \frac{d}{d y} f(y, \lambda)\right] \\
+\mu(\lambda) \phi\left(y \sqrt{1+\lambda^{2}}\right) \sum_{i=1}^{k} \frac{(2 k-1) ! !}{(2 i-1) ! !} \sum_{j=1}^{i} \frac{(2 i-2) ! !}{(2 j-2) ! !} \frac{\left[(2 j-2) y^{2 i-3}-\left(1+\lambda^{2}\right) y^{2 i-1}\right]}{\left[1+\lambda^{2}\right]^{i-j+1}} .
\end{gathered}
$$


Now, it is easy to see that:

$$
\sum_{j=1}^{i} \frac{(2 i-2) ! !}{(2 j-2) ! !} \frac{\left[(2 j-2) y^{2 i-3}-\left(1+\lambda^{2}\right) y^{2 i-1}\right]}{\left[1+\lambda^{2}\right]^{i-j+1}}=-\sqrt{1+\lambda^{2}} y^{2 i-1}
$$

Hence (3.10) reduces, after simplification, to:

$$
\begin{gathered}
f(y, \lambda) \sum_{i=1}^{k} \frac{(2 k-1) ! !}{(2 i-1) ! !} y^{2 i}+\left[\frac{d}{d y} f(y, \lambda)\right] \sum_{i=1}^{k} \frac{(2 k-1) ! !}{(2 i-1) ! !} y^{2 i-1}= \\
\mu(\lambda) \sqrt{1+\lambda^{2}} \phi\left(y \sqrt{1+\lambda^{2}}\right) \sum_{i=1}^{k} \frac{(2 k-1) ! !}{(2 i-1) ! !} y^{2 i-1}
\end{gathered}
$$

Or,

$$
\left[\frac{d}{d y} f(y, \lambda)+y f(y, \lambda)\right] \sum_{i=1}^{k} \frac{y^{2 i-1}}{(2 i-1) ! !}=\mu(\lambda) \sqrt{1+\lambda^{2}} \phi\left(y \sqrt{1+\lambda^{2}}\right) \sum_{i=1}^{k} \frac{y^{2 i-1}}{(2 i-1) ! !}
$$

Finally, since $\sum_{i=1}^{k} \frac{y^{2 i-1}}{(2 i-1) ! !}$ is a polynomial in y of degree (2k-1) with rational coefficients, then (3.11) reduces:

$$
\frac{d}{d y} f(y, \lambda)+y f(y, \lambda)=\mu(\lambda) \sqrt{2 \pi} \sqrt{1+\lambda^{2}} \phi(y) \phi(\lambda y) .
$$

It is easy to see that this first order differential equation in the unknown function $\mathrm{f}(\mathrm{y})$ has the solution given by (3.4). Therefore $X \sim \mathrm{SN}(\lambda)$. This completes the proof of Theorem 2.5 .

\section{Proof of Theorem 2.6:}

The proof of this theorem is similar to that of Theorem 2.5.

\section{Specialization to the Standard Normal Distribution}

As the skew-normal distribution tends to the standard normal distribution for $\lambda=0, \quad$ then putting $\lambda=0$, theorems 2.1-2.4, will be valid for the standard normal distribution and can be restated as follows; keeping all the rotations used there:

\section{Theorem 4.1}

Let $X$ be a continuous rv with pdf $f($.$) , cdf F($.$) , survival function \bar{F}($.$) , failure rate h($.$) and let \mathrm{X}_{1: \mathrm{n}} \leq \ldots \leq$ $\mathrm{X}_{\mathrm{n}: \mathrm{n}}$ be the os of a random sample of size $n$ drawn from $X$. Suppose that $F($.$) has a continuous second order$ derivative on $(-\infty, \infty)$. Then $X$ has the standard normal distribution if and only if:

$$
E\left(X_{k+1: n}+\cdots+X_{n: n} \mid X_{k: n}=y\right)=(n-k) h_{0}(y) .
$$

\section{Theorem 4.2}

Let $X$ be a continuous rv with pdf $f($.$) , cdf F($.$) and let \mathrm{X}_{1: \mathrm{n}} \leq \mathrm{X}_{2: \mathrm{n}} \leq \ldots . \leq \mathrm{X}_{\mathrm{n}: \mathrm{n}}$ be the os of a random sample of size $n$ drawn from $X$. Suppose that $F($. $)$ has a continuous second order derivative on $(-\infty, \infty)$. Then $X$ has the standard normal istribution if and only if:

$$
E\left(X_{1: n}+\cdots+X_{k-1: n} \mid X_{k: n}=y\right)=-\frac{(k-1)}{F_{0}(y)} f_{0}(y) .
$$

\section{Theorem 4.3}

Let $X$ be a continuous rv with pdf $f($.$) , cdf F($.$) , survival function \bar{F}($.$) , failure rate h($.$) and let \mathrm{X}_{1: \mathrm{n}} \leq \mathrm{X}_{2: \mathrm{n}}$ $\leq \ldots \leq \mathrm{X}_{\mathrm{nn}}$ be the os of a random sample of size $n$ drawn from $X$. Suppose that $\mathrm{F}($.) has a continuous second order derivative on $(-\infty, \infty)$. Then $X$ has the standard normal distribution if and only if:

$$
E\left(S_{k}-\bar{S}_{k} \mid X_{k: n}=y\right)=y-m h_{0}(y)\left[F_{0}(y)\right]^{-1} .
$$

\section{Theorem 4.4}

Let $X$ be a continuous rv with pdf $f($.$) , cdf F($.$) , survival function \bar{F}($.$) , failure rate h($.$) and finite mean \mu$ 
and let $\mathrm{X}_{1: \mathrm{n}} \leq \mathrm{X}_{2: \mathrm{n}} \leq \ldots . \mathrm{X}_{\mathrm{n:n}}$ be the os of a random sample of size $n$ drawn from $X$. Let $F($.$) has a continuous$ second order derivative on $(-\infty, \infty)$. Then $X$ has the standard normal distribution if and only if:

$$
E\left(T_{k} \mid X_{k: n}=y\right)=y+\frac{m}{F_{0}(y)} h_{0}(y)\left[2 F_{0}(y)-1\right],
$$

where $\mathrm{n}=2 \mathrm{~m}+1, \mathrm{k}=\mathrm{m}+1$ and $\mathrm{m}=1,2,3, \ldots$.

\section{Theorem 4.5}

Let $X$ be a continuous rv with pdf $f($.$) , cdf F($.$) , survival function \bar{F}($.$) and failure rate h($.$) . Assume that$ $\mathrm{E}\left(\mathrm{X}_{\mathrm{U}(\mathrm{n}+1)}\right)$ is finite. Then $X$ has the standard normal distribution if and only if:

$$
E\left(X_{U(n+1)}^{2 k} \mid X_{U(n)}=y\right)=(2 k-1) ! !+h_{0}(y) \sum_{i=1}^{k} \frac{(2 k-1) ! !}{(2 i-1) ! !} y^{2 i-1}, \quad k=1,2,3, \ldots
$$

\section{Theorem 4.6}

Let $X$ be a continuous rv with pdf pdf $f($.$) , cdf \mathrm{F}($.), survival function $\bar{F}($.$) and failure rate h($.$) . Assume$ that $\mathrm{E}\left(\mathrm{X}_{\mathrm{U}(\mathrm{n}+1)}\right)$ is finite. Then $X$ has the standard normal distribution if and only if:

$$
E\left(X_{U(n+1)}^{2 k+1} \mid X_{U(n)}=y\right)=h_{0}(y) \sum_{i=0}^{k} \frac{(2 k) ! !}{(2 i) ! !} y^{2 i}, \quad k=0,1,2, \ldots
$$

\section{Conclusion}

In this study, some characterization results for the skew-normal distribution are proved based on the conditional moments of order statistics as well as the conditional moments of record values of a random sample drawn from this distribution. Special cases regarding the standard normal distribution are also given. The achieved results are of direct relevance to fitting and modeling skew data using the skew-normal distribution. Moreover, the new results are more appropriate for practical applications comparing with the results of Gupta et al. (2004) which is based on distribution properties. Also, our results motivate the researchers toward a serious consideration of other characterization problems for the skew-normal distribution such as characterization using regression properties and residual life time. Further, the future research challenge is how to extend the present results to the case of the multivariate skew-normal distribution. The limitations is summarized in that it is applied only for the skew-normal distribution and its specialized distribution.

\section{Acknowledgement}

The authors are greatly indebted to the referees and the editors for their constructive comments.

\section{References}

Ahsanullah, M. (1995). Record statistics. Nova Science Publishers, New York.

Ahsanullah, M. (2004). Record values. Theory and Applications. University press of America, New York.

Ahsanullah, M. \& Ragab, M. Z. (2006). Bounds and characterization of Record statistics. Nova Science Publishers, Hauppauge, New York.

Arfken, G. (1985). Mathematical methods for physicists, 3rd ed. Orlando, FL: Academic Press.

Arnold, B. C., Balakrishnan, N. \& Nagarajua, H. N. (1998). Records. John Wiely and Sons, New York. http://dx.doi.org/10.1002/9781118150412

Arnold, B. C., Balakrishnan, N. \& Nagarajua, H. N. (2008). A First Course in Order Statistics. 1st Edn., Wiely, New York. http://dx.doi.org/10.1137/1.9780898719062

Azzalini, A. (1985). A class of distributions which includes the normal ones. Scand J. Stat., 12, 171-178.

Azzalini, A. (1986). Further results on a class of distributions which includes the normal ones. Statistical Papers, 46, 199-208.

Brown, N.D. (2001). Reliability studies of the skew-normal distribution. Thesis, Graduate School, Maine University. USA.

Gharib, M., Salehi, S. \& Sharawy, A. (2014). Characterizations of the skew-normal distribution Via characteristic function and conditional moments. J. Advanced Research in Applied Math., 6, 10-22. 
http://dx.doi.org/10.5373/jaram.1838.090513

Gupta \& Wen-Jang, H. (2002). Quadratic forms inskew normal variates. J. Math. Anal. Appl., 273, 558-564. http://dx.doi.org/10.1016/S0022-247X(02)00270-6

Gupta \& Jose, T. (2004). Characterization of the skew normal distribution. Ann. Inst. Statist. Math., 2, $351-360$. http://dx.doi.org/10.1007/BF02530549

Shawky, A. I., \& Abu-Zinadah, H. H. (2006). General recurrence relations and characterizations of certain distributions based on record values. J. of Approximation Theory and Applications, 2, 149-159.

Shawky, A. I., \& Abu-Zinadah, H. H (2008b). General recurrence relations and characterizations of certain distributions based on record statistics. J. of Stat. Theory and Applications, 7, 93-117.

Shawky, A. I., \& Bakoban, R. A (2008). Characterization from exponentiated gamma distributions based on record values. J. of Stat. Theory and Applications, 7, 263-277.

\section{Copyrights}

Copyright for this article is retained by the author(s), with first publication rights granted to the journal.

This is an open-access article distributed under the terms and conditions of the Creative Commons Attribution license (http://creativecommons.org/licenses/by/3.0/). 